\title{
Las compañías extranjeras en la tecnificación de la ciudad a comienzos del siglo XX
}

\section{Foreign companies in the technification of the city at the beginning of the 20th century}

\section{Carolina Salazar-Marulanda*}

\section{Resumen}

El presente trabajo enmarca el proceso de tecnificación de las ciudades a partir del planeamiento y diseño de obras públicas para tender redes de agua y sanear las infraestructuras urbanas en los inicios del siglo XX. Desde los adelantos conseguidos en la Revolución Industrial y la transformación técnica de los procesos por medio de nuevos materiales y procesos científicos, los profesionales se alejaron de los sistemas artesanales para incorporar normativas y herramientas en la búsqueda de ambientes saludables, libres de epidemias y enfermedades. Así, los avances alcanzados por la ciencia fueron aplicados por compañías europeas y norteamericanas que, mediante el desarrollo de infraestructuras enfocadas en conseguir productividad, eficiencia y comodidad, tomaron la ciudad como escenario para introducir masivamente tecnologías y herramientas de vanguardia. De esta manera, la compañía inglesa Pearson $\&$ Son evidencia la intervención extranjera como el medio a través del cual se desarrollaron las redes de servicios públicos, que desencadenaron los mayores procesos de urbanización en las primeras décadas del siglo XX.

Palabras clave: Siglo XX; Higienización; Ingenieros; Obra pública; Redes de servicios.

\begin{abstract}
This work frames the process of technification of cities from the planning and design of public works to build waternetworks and clean up urban infrastructures in the early twentieth century. From the advances made in the Industrial Revolution and the technical transformation of processes through new materials and scientific processes, professionals moved away from artisanal systems to incorporate regulations and tools in the search for healthy environments, free of epidemics and diseases. Thus, the advances achieved by science were applied by European and North American companies that through the development of infrastructures focused on achieving productivity, efficiency and comfort, took the city as a stage to massively introduce cutting-edge technologies, and tools. In this scenario, the British company Pearson \& Son evidences the foreign intervention as the means through which the public service networks that unleashed the greatest urbanization processes in the first decades of the 20th century were developed.
\end{abstract}

Keywords: 20th Century; sanitation; engineers; public works; service networks.

* Profesora Asociada en la Universidad Nacional de Colombia. Arquitecta (Universidad Nacional de Colombia, Manizales, 1997), magister en Historia de la Arquitectura (Universidad Nacional de Colombia, Bogotá, 2013), candidata a Doctora en Historia'(Universidad Nacional de Colombia, Medellín).E-mail: csalazarma@unal.edu.co 


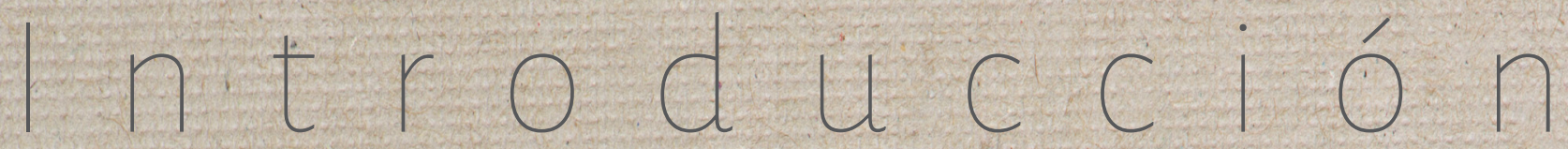

a ciudad puede observarse como un laboratorio de experimentación en el que concurren variedad de actores, símbolos, flujos, herramientas e intercambios; lugar donde se representan los conceptos de público y privado, individual y colectivo de manera simultánea y complementaria. A partir de la Segunda Revolución Industrial, la estructura urbana se convirtió en un escenario industrializado, asumiendo los conceptos de progreso y modernidad a través de la introducción de saberes científicos e intervenciones, que por sus características, podrían denominarse como Big Science'. Estas transformaciones buscaban engrandecer la imagen de los Estados², entendiendo la dignificación de las ciudades como representación de prosperidad y convergencia de saberes científicos y técnicos, por lo que en su mayoría estuvieron lideradas por ingenieros y médicos de profesión.

Esta necesidad de construir infraestructura, que reflejara la llegada del progreso, hizo que las labores del ingeniero estuvieran asociadas a los planes de gobierno que proyectaban implementar los avances de la técnica en beneficio del saneamiento de las ciudades y el desarrollo del comercio. Así, adaptando los principios de la ciencia a las necesidades de cada lugar, se plantearon proyectos para mejorar los sistemas de comunicación por medio de la construcción de ferrocarriles y carreteras; se buscó el mejoramiento de las condiciones de navegabilidad de los ríos; se aprovecharon los recursos naturales para la producción de energía, facilitando la iluminación urbana y la implementación de sistemas de transportey maquinaria industrial; y se construyeron edificios acondicionados

Este nuevo poder asociado al conocimiento, convirtió los científicos en personajes públicos y llevó a los políticos a rodearse de sabios y técnicos, en una constante emulación a Francia e
Inglaterra como símbolos de
modernidad.

1 El término Big Science haciendo referencia a "la escala de las operaciones, el número de actores implicados, las repercusiones públicas y la pluralidad de las tecnologías utilizadas" en las intervenciones urbanas realizadas a partir de la segunda mitad del siglo XIX (LAFUENTE, 2001).

2 "El discurso que sostiene el proceso de formación del Estado se resume en la palabra progreso [...] y progreso y tecnología parecían una misma cosa" (LAFUENTE, 2001). 
para albergar higienicamente funciones relativas al cuidado de la salud y el manejo de alimentos, poniendo la profesión al servicio de la Nación en la búsqueda por avanzar haciá la modernización de las ciudades (VARIAS..., 1916, p.113-117).

En las postrimerías del siglo XIX y las primeras décadas del XX, la transformación urbana, la industrialización económica y la centralización estatal, resumieron su discurso en la palabra progreso. Este nuevo poder asociado al conocimiento, convirtió los científicos en personajes públicos y llevó a los políticos a rodearse de sabios y técnicos, en una constante emulación a Francia e Inglaterra como símbolos de modernidad. Se trajeron a América los adelantos presentados en exposiciones industriales referidos a ferrocarriles, arquitectura de hierro, innovaciones técnicas y de higiene urbana, por medio de los cuales, se acercaba y ponía la ciencia al servicio urbano (LAFUENTE, 2001).

En este escenario modernizador, los ingenieros tuvieron amplia participación en diversos círculos de poder, lo cual permitió la dinamización y el avance de la cultura educativa y material. La migración de profesionales significó el trasvase de conocimientos y la divulgación de técnicas y productos no solo a través del trabajo, sino del incremento de publicaciones especializadas y la difusión de los últimos adelantos conseguida por medio de exposiciones universales. De esta manera, aunque hasta finales del siglo XIX habían sido los médicos los encargados de generar y aplicar las normas del higienismo ${ }^{3}$, en los inicios del siglo XX fueron los ingenieros ${ }^{4}$ quienes lideraron el diseño y la construcción de infraestructuras para producir ambientes urbanos sanos, y en este sentido, los proyectos desarrollados en Latinoamérica por compañías europeas, no solo contribuyeron a la higienización de las ciudades, sino que propiciaron la homogeneización de la cultura tecnológica y del mercado.

Así, con la optimización del modelo urbano por medio de la aplicación de la técnica, se introdujeron los conceptos de orden, saneamiento y control social en las ciudades latinoamericanas, tomando las obras públicas como reflejo del progreso y cónvirtiéndolas en el hilo conductor de las reformas aplicadas en favor de la salubridad urbana. Las compañías extranjeras, y en especial, los avances de la ingeniería británica en proyectos para abastecimiento y tratamiento de agua en la Europa de ultramar, se reflejan en el trabajo de la casa Pearson \& Son, la cual llegó a construir en las primeras décadas del siglo XX importantes obras de saneamiento en países como Estados Unidos, México, Chile y Colombia.

3 El higienismo deja de entenderse como un concepto de salud relativo a la medicina, para convertirse en una serie de normativas aplicadas por abogados, ingenieros y administrativos pertenecientes a organismos gubernamentales, con el fin de reglamentar los procesos de desarrollo y crecimiento de las ciudades.

4 Los ingenieros de finales del siglo XIX y comienzos del siglo XX, se convierten en los intermediarios entre los intereses estatales, la materialización de los proyectos y el beneficio social, destacándose social, política y económicamente como garantes en proyectos para el diseño y construcción de sistemas de comunicación y obras públicas. 


\section{El agua como símbolo de podery ordenamiento urbano}

Tanto en Europa como en América, uno de los primeros elementos que sirvió para establecer un orden social en las ciudades fue el acceso al agua. En efecto, los últimos años del siglo XIX se caracterizaron por una problemática ambiental que comprometía los estándares mínimos de higiene requeridos para la vida en comunidad y que se relacionaban directamente con el manejo de las aguas en los conglomerados urbanos. Esta situación se extendió incluso en Europa hasta 1950 (CAPEL, 2000), pues solo en ese momento, se generalizó la cobertura del servicio de agua en todos los estratos sociales. Para el continente americano, esta temporalidad resulta muy similar, ya que fue, durante las primeras décadas del siglo $X X$, que las ciudades iniciaron un proceso de modernización acorde a la consolidación de las naciones y al acceso a los mercados internacionales. En este sentido, la construcción de infraestructuras de servicios públicos ${ }^{5}$ en Europa y América se desarrolló en medio del traslado de ingenieros profesionales que, a través de su trabajo, unificaron los procesos e incorporaron la técnica en las obras, generándose un avance simultáneo en la dotación de redes de servicios en el antiguo y el nuevo continente.

Con relación a Europa, una de las primeras naciones en preocuparse por la prestación de servicios públicos para mejorar las condiciones de salud en las ciudades fue Gran Bretaña ${ }^{6}$. Valiéndose de los avances técnicos, se generaron a lo largo del siglo XIX mecanismos para conducir y evacuar las aguas hasta y hacia los barrios obreros, procurando una solución a los problemas higiénicos relativos a la concentración masiva de trabajadores en los conglomerados industriales?. Esta situación le dio a la nación británica cierta ventaja en la instalación de servicios relativos al saneamiento urbano, lo cual representaría más adelante, para los ingenieros ingleses, participación y reconocimiento en proyectos de este tipo a nivel mundial.

Durante este mismo periodo, en la mayoría de ciudades europeas, no existía aún un sistema de saneamiento de aguas residuales y comúnmente se arrojaban todos los desperdicios a los ríos, siendo solo hasta finales del siglo que se empezaron a incorporar redes de alcantarillado

5 No es casual que Antonio Pedrini presentase en 1905 un trabajo titulado La cittá moderna como compendio de las bondades sociales de la ingeniería sanitaria y de la técnica, claves que consideraba como auténticas impulsoras de las mejoras de la urbe (RUEDA, 1998, p. 179).

6 El filósofo y jurisconsulto británico Jeremy Bentham ejerció una fuerte influencia sobre la higiene pública con su ideología utilitarista, siendo una de las personalidades que más contribuyeron a la formación del pensamiento constitucionalista europeo de principios del siglo XIX (ALCAIDE, 1999).

7 Sanitary movement no era sólo una cuestión técnica de infraestructuras de alcantarillado y agua corriente, sino que a través de la mejora del ambiente urbano se pretendía prioritariamente la estabilidad social y la moralización de las clases obreras, además de la consecución de ciertos beneficios económicos (RAMOS, 2014). 
en los planes de saneamiento y expansión urbana:. Esta situación, que se tornaba inmanejable por el aumento de habitantes en las ciudades, empezó a controlarse con la implementación de mecanismos que conducían el agua a presión por tuberías hasta las viviendas y posteriormente evacuaban los desechos, valiéndose de corriente hidráulica y gravedad a través de las alcantarillas. Solo a partir de este momento, puede hablarse de las redes de servicios como un sistema de distribución general del agua para toda la ciudads.

Sin embargo, la construcción de estas redes no representaba una prestación pública del servicio. El acceso al agua era cuestión de ricos; para los pobres, las condiciones seguían siendo problemáticas con relación a la obtención del líquido. Hasta 1850, el agua potable era vendida a los pobladores que podían comprarla, los demás debían tomarla de las fuentes públicas con todos los problemas higiénicos que ello representaba. Así mismo, en las zonas donde aún se carecía de alcantarillado, las aguas residuales eran depositadas en letrinas que debían ser vaciadas regularmente, lo cual también representaba contar con los recursos económicos para poder pagarlo.

En lo que se refiere a América el caso era semejante. Las ciudades coloniales carecían de redes de acueducto y alcantarillado y, hasta finales del siglo XIX, el agua potable debía ser tomada de las fuentes y aljibes dispuestos en plazas o lugares comunes para el abastecimiento de la población. Igualmente, las aguas residuales corrían por las acequias construidas en medio de las calles, transportando a cielo abierto toda clase de desechos. Esta situación, comenzó a transformarse radicalmente finalizando la centuria del diecinueve, con la construcción de ciudades modernas y planificadas, que garantizaban urbes higiénicas en las cuales el ciclo del agua se completaba de manera integral ${ }^{10}$. Los casos de Chicago en Estados Unidos (1871), La Plata en Argentina (1882) y Belo Horizonte en Brasil (1897) son, entre otros, ejemplos que demuestran la importancia de las redes como factor de desarrollo en el planeamiento y expansión de la ciudad.

Sin embargo, el caso de las nuevas ciudades no era comúny la mayoría de las existentes en ambos continentes debía enfrentar el incremento demográfico, manteniendo su antigua estructura ${ }^{11}$, lo

8 Hasta muy avanzado el siglo XIX en las ciudades españolas era muy común la utilización de pozos negros para la evacuación de aguas residuales, ya que la ausencia de cloacas se convirtió en un serio problema, al impedir el desagüe de una manera higiénica y saludable. Una de las primeras soluciones para convertir el espacio urbano en un lugar libre de epidemias y sectores insalubres fue utilizar el rio Manzanares para evacuar los desechos, condición que empeoraría, al popularizarse el sistema de saneamiento "tout à l'egout" y que solo empezaría a mejorar finalizando el siglo con la construcción de alcantarillados (CAPEL, 2000).

9."En un periodo relativamente corto en relación con la historia, las ciudades se han dotado de ló que hoy llamamos redes [...] introduciendo progresiva pero continuamente, un conjunto de dispositivos basados en principios técnicos variados" (DUPUY, 1998, p. 35-36).

$10 \mathrm{El}$ ciclo del agua se refiere al proceso de ábastecimiento de agua directamente en las viviendas, por medio de tuberías conectadas al acueducto y al sistema complementario de alcantarillas a través de las cuales se desalojan las aguas residuales y los desperdicios utilizando canales subterráneos.

11 En el caso de Colombia, la población se incrementó drásticamente en un periodo de sesenta años, pasando de 2.4 millones de habitantes en 1870 a 4 millones en 1900 y alcanzando los 8 millones en 1930 (SUÁREZ; MONSALVÖ, 2013, p. 101). 
cual hacía más complicado alcanzar los estándares de calidad relacionados con la introducción a la vida urbana de los conceptos del higienismo. Esta preocupación por la higiene surgió a partir del deseo de las elites de conseguir entornos saludables alejados de los focos epidémicos e insalubres que caracterizaban las ciudades industriales de finales del siglo $X X^{12}$. En este sentido, las campañas buscaban establecer códigos sanitarios para evitar el contagio y propagación de enfermedades; imponer estándares de calidad en el manejo y distribución de alimentos; crear instituciones y órganos de difusión para el cuidado de la salud pública; tender redes de servicios que garantizaran cobertura y buen manejo de las aguas; y fomentar entre la población la adopción de habitos de limpieza y habitabilidad de las edificaciones (SUÁREZ; MONSALVO, 2013, p. 102).

Esta necesidad de combatir las enfermedades, las epidemias, el hacinamiento y la mortalidad implicaba no solo un cambio de mentalidad y de comportamiento, sino la implementación de infraestructuras idóneas, órganos de difusión y el mejoramiento de instalaciones relativas a la modernización de la ciudad ${ }^{13}$. De ahí que fuera imposible el saneamiento de las poblaciones sin contar con redes de servicios públicos que garantizaran un abastecimiento continuo de agua limpia, la construcción de una red de cloacas para las evacuaciones y la reglamentación de la higiene pública que garantizara la protección de la salud de los habitantes (CASAS, 2000, p. 349-377).

Con la concientización general sobre la importancia de la incorporación de los conceptos del higienismo para garantizar ciudades saludables, y gracias a la implementación de técnicas para facilitar el acceso al agua, los hábitos cotidianos también se modificaron, convirtiendo la limpieza en un nuevo valor social ${ }^{14}$. Ante la ausencia de cuartos de baño domésticos, las clases altas que contaban con poder económico popularizaron la utilización de espacios comunes que les permitieran distinguirse socialmente por medio del aseo corporal. Esta nueva forma de sociabilidad, hizo que las casas de baño se convirtieran en el escenario para mantener la salud y reconocerse socialmente como individuos de comportamiento civilizado y moderno, asimilando las normas de higiene como un beneficio de sanidad y de distinción social. Estos espacios habían estado presentes en la vida urbana con anterioridad's, pero fue a lo largo del siglo XIX que su utilización vino a representar un cumplimiento de estándares asociados al

12 La elite, promotora de esta nueva forma de vida urbana, estaba integrada por miembros que eran promotores de la industrialización y consideraban la verdad científica como el camino para alcanzar el desarrollo (HENDERSON, 2006, p. 107). 13 El urbanismo europeo buscaba instrumentos analíticos, proyectuales, normativos y legislativos entendidos como base del proyecto: apareció la zonificación; la división en lotes; se regularizaron las tipologías, se definieron pautas de alineaciones, normas de higiene y ordenanzas anti-incendio etc., codificándose un saber al que técnicos y administrativos podían recurrir para definir la organización de la ciudad (SAMBRICIO, 2017, p. 206).

14 "En el último tramo del siglo XIX, se intenșificaron, las acciones que permitirían conseguir la universal consagración de la limpieza corporal como un valor social, instalando la idea de que el baño era un hábito necesario para el mantenimiento de la salud y de un nuevo 'orden' social" (VALLEJO, 1997, p. 58).

15 En las grandes concentraciones urbanàs de la sociedad industrial, se hizo evidente la necesidad de contar con espacios que facilitaran el mantenimiento de estándares mínimos de aseo0 (VALLEJO, 1997, p. 60). 
prestigio sociali6. Sin embargo, la población que no podía pagar por este servicio debía recurrir a los ríos o vivir sin asearse, situación que muy pronto comprometió la salubridad colectiva y puso de manifiesto la implementación de la higiene pública más allá de considerar el acceso al agua como un beneficio particular y suntuoso.

En este sentido, además de las obras relacionadas con el manejo del agua en las ciudades, también en las viviendas empezaron a incorporarse elementos que no solo representaban una vida más saludable, sino de confort y bienestar. Sí la electricidad permitió prolongar el día e introdujo nuevas actividades para desarrollar en la noche, el agua produjo una variación de la tipología urbana y doméstica, que conllevó la aparición de nuevos espacios y áreas acondicionadas con equipamientos que permitieron su uso controlado y adecuado. La introducción de cuartos de baño, instalaciones de cocina, aparatos y accesorios transformó las viviendas y llevó poco a poco a la optimización del suministro de agua domiciliaria.

Aunque el acceso al agua en los centros urbanos estuvo ligado al poder económico y se generaron zonas degradadas por la falta del servicio, durante la primera mitad del siglo XX las redes de acueducto y alcantarillado alcanzaron una cobertura más acorde a la expansión de las ciudades, presentándose como un elemento esencial para regularizar, embellecer y sanear las estructuras urbanas. Con el agua se introdujeron nuevas formas de civilidad, representadas en novedosas infraestructuras y en la habilidad de los ingenieros (CASAS, 2000, p. 349-377) para traducir a fórmulas técnicas las contradicciones sociales y culturales aparejadas al crecimiento demográfico (LAFUENTE, 2001), elevando el papel de estos profesionales al mayor protagonismo en la tecnificación de la ciudad durante este periodo.

\section{La higienización y las compañías extranjeras}

Equipar las ciudades con redes de abastecimiento y evacuación de agua marcó una diferencia espacial significativa, no solo por la implementación domiciliaria del servicio y las comodidades relacionadas con su disfrute, sino por la transformación asociada al cambio de hábitos ligados a la higienización y creación de espacios saludables. Si bien en la agenda de los gobernantes se implementaron diversas medidas para afrontar el crecimiento urbano y demográfico de principios del siglo XX, fueron las redes de servicios (DUPUY, 1998, p. 35-36) las que impusieron un criterio de ordenamiento y crecimiento que terminó determinando la zonificación y estratificación de las

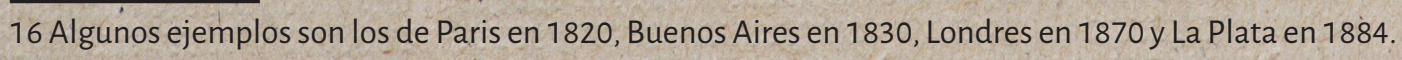


ciudades, generando áreas de expansión y exclusión que condicionaron los usos urbanos en un periodo de tiempo relativamente muy corto ${ }^{17}$.

Las redes de servicios actuaron como elementos ordenadores de la estructura y los procesos urbanos, permitiendo articular las nuevas funciones, relaciones y espacios en la expansión de la ciudad (FRISCHMANN, 2012, p. XI). Sin embargo, aunque la disponibilidad de agua para suplir las principales necesidades humanas en los conglomerados urbanos era fundamental, fueron los procesos correctos de distribución y recolección los que se convirtieron en factores de desarrollo y progreso, siendo fundamental la implementación de la técnica y la dirección de los procesos por parte de profesionales expertos, lo cual llevó a que el Estado optara, en la mayoría de los casos, por contratar los servicios de compañías extranjeras (SANÍN, 1927, p. 633).

Así, una de las principales compañías que desarrolló obras de saneamiento, distribución y evacuación de agua en varios países de América fue Pearson and Son, empresa conformada en Bradford (Inglaterra) por Samuel Pearson (1814-1884) en el año 1844 y que en sus comienzos se dedicaba a la fabricación de ladriIlos, baldosas y tuberías sanitarias. Posteriormente adoptó el nombre de S. Pearson and Son, cuando su hijo Ceorge ingresó al negocio en 1856. Rápidamente las actividades de la empresa se diversificaron desarrollando líneas ferroviarias, redes para suministro de agua, drenaje y alcantarillado en ciudades industriales en expansión. Ante el retiro de su abuelo Samuel, el ingeniero Weetman Pearson asumió la dirección de la compañía en 1879, convirtiéndose más tarde en el único propietario del conglomerado (PEARSON PLC).

En la década de 1880 la compañía se posicionó en el escenario internacional desarrollando obras como puertos, ferrocarriles, túneles, suministro de agua y drenaje. En 1881 inició la cons-

17 En Colombia la mayoría de ciudades capitales de departamento tendieron sus redes de acueducto y alcantarillado durante las priméras tres décadas del siglo XX. 
trucción del Muelle de Bentinck en Lynn, y en 1884 se trasladaron las oficinas a Londres, desde donde desarrollaron obras en Europa, Estados Unidos, Canadá y América Latina. Durante este mismo periodo, se emprendió la construcción de muelles como Milford Haven (1885-1890), Southampton (1886-1991) y Halifax, Nova Scotia (1886-1889), especializándose en el manejo de obras hidráulicas. Esta condición significó la adjudicación de importantes contratos, como la construcción del túnel del río Hudson en Estados Unidos y el Gran Canal para drenar los pantanos de la Ciudad de México, ambos proyectos construidos en 1889.

Entre 1891 y 1897 trabajaron en la construcción del túnel Blackwall bajo el río Támesis, empleando por primera vez un escudo neumático para su excavación. Gracias a estas obras, la compañía Pearson aseguró su renombre a nivel mundial, desarrollando trabajos de saneamiento y tratamiento de aguas a gran escala. En 1900 los contratistas británicos se encargaron de la construcción de los túneles bajo el East River que conectan New York con Long Island para Pennsylvania, New York y Long Island Railroad Company, ante lo cual se conformó la filial estadounidense S. Pearson and Son Inc.

Foto 1: Excavación neumática bajo el río Tamesis.

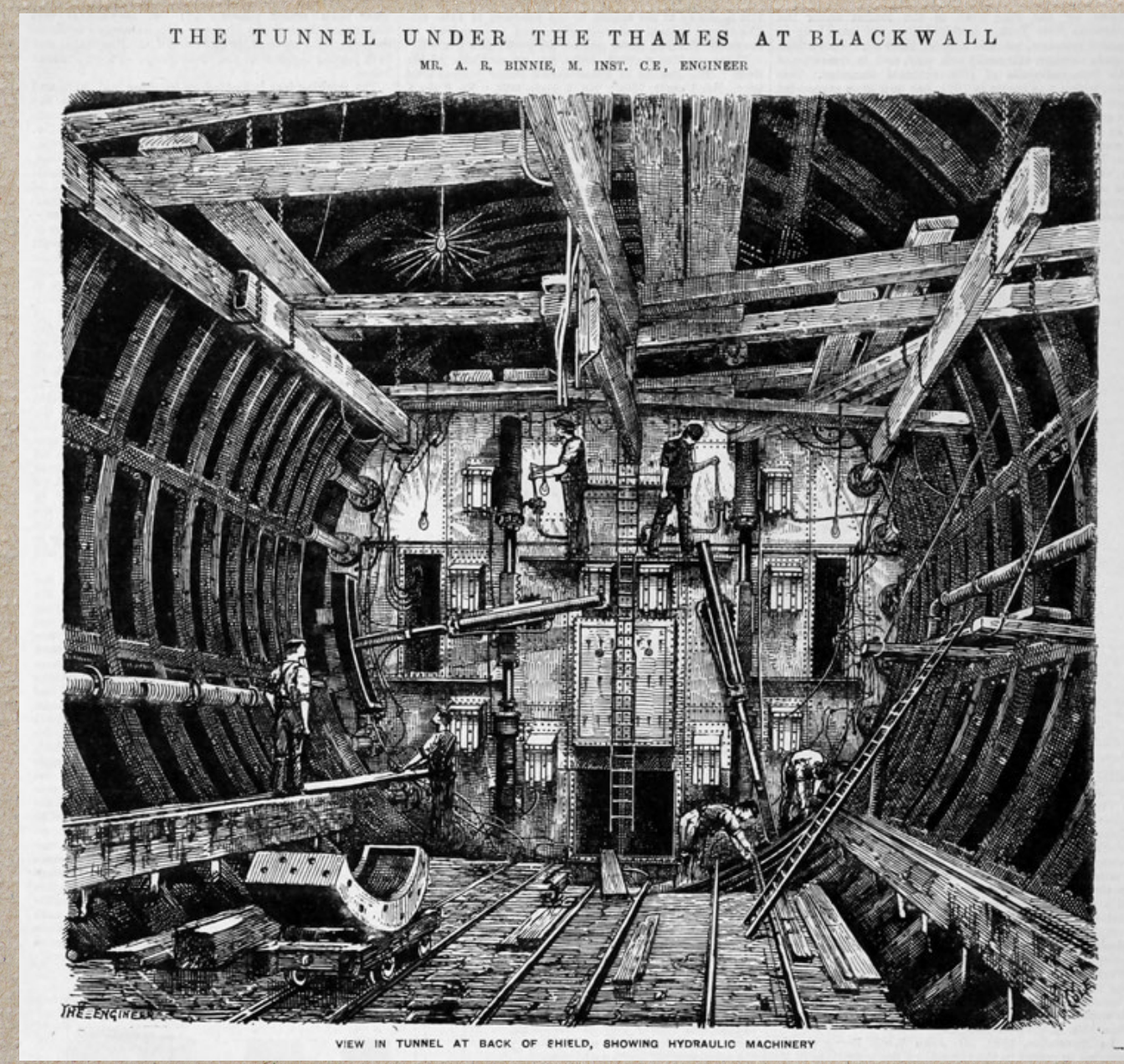

Fuente: Pearson and Son (1895). 
En adelante, los británicos comenzaron a adquirir concesiones petroleras tras el descubrimiento de filtraciones de petróleo en México durante una de las excavaciones, motivo que los Ilevó, en 1903, a establecerse en las montañas de Puebla, en donde terminaron la construcción del Great Northern and City Railway en 1904, comprometiéndose con su operación durante tres años. Sin embargo, estos trabajos llevarían a la empresa a enfrentar la oposición de los mexicanos por los términos establecidos en la realización del contrato ${ }^{18} y$, más adelante, sus concesiones petroleras pasarían a Mexican Eagle Petroleum Co. en 1909.

S. Pearson and Son se caracterizó por la diversificación de sus actividades. Además de los trabajos hidráulicos, incorporó después de 1900 la generación, suministro y aplicación de energía eléctrica en América Latina, comenzando por el sistema de tranvías y ampliándose posteriormente al suministro de electricidad en Veracruz y otros lugares de México. Este servicio también se desarrolló en Chile después de la Primera Guerra Mundial, conformándose la Pearson's Cia. Chilena de Electricidad.

Ya en Chile, la compañía realizó en Valparaíso las obras para la construcción del puerto, aportando no solo la técnica para enfrentar la primera mega obra del país, sino la posibilidad de ganarle terreno al mar en una nación con grandes limitaciones de área. El primer contrato se desarrolló entre 1912 y 1923 y el segundo entre 1923 y 1930. El 6 de octubre de 1912 la compañía inició los trabajos que habían sido definidos desde 1910 por la Junta Central de Puertos y que se dividieron en tres etapas.

La primera consistía en levantar el rompeolas que partía desde Punta Duprat en dirección al oriente, prolongándose por 288 metros, un malecón de 12 metros de alto por 630 metros de largo, sobre profundidades de 55 metros. Se concretó también un espigón de 250 metros de largo y un muelle para descarga de carbón. (EL INGENIERO..., 2013, p. 7)

Así, los ingleses llevaron a cabo un proyecto de grandes proporciones con el cual dieron solución al manejo del puerto y las condiciones ambientales que afectaban su funcionamiento. Igualmente, contribuyeron al desarrollo de la ingeniería nacional al encargar de la obra al ingeniero civil chileno Eduardo Budge, quien actuó como representante de la empresa constructora de Londres y jefe de las obras portuarias, lo cual condujo al intercambio de conocimientos y la capacitación de ingenieros locales para la aplicación de la técnica importada por los británicos en otras obras públicas. Aunque esta obra fue proyectada para siete años, tuvo que pro-

18 En la colección "Documentos Porfiristas" de la Biblioteca Aportación Histórica, se publica en 1948 un documento titulado "La concesión del ferrocarril de Tehuantepec a la Pearson and Son Limited de Londres", en el cual se expone la crítica aparecida en el periódico de oposición al gobierno de Porfirio Díaz, El Correo de México, del 6 de octubre de 1906 y que reseña las desventajas en la adjudicación de aportes para, la construcción y porcentajes de explotación entre el gobierno y el "grupo científico" (LA CONCESIÓN..., 1906). 
longarse por siete más, debido a la falta de materiales derivada de la Primera Guerra Mundial, situación que obligó a detener las obras.

\section{El proyecto de saneamiento de Bogotá}

La casa Pearson $\&$ Son elaboró para Bogotá, en 1912, un proyecto de saneamiento consistente en la implementación de alcantarillas que permitieran evacuar las aguas Iluvias de la ciudad, la cual, para entonces, aún conservaba el sistema colonial que las dejaba correr superficialmente. El proyecto elaborado por el ingeniero consultor Sir W. Fox M. planteaba un sistema fuertemente criticado por los ingenieros colombianos Alberto Borda Tanco, Pedro Uribe y Julio Garavito, quienes consideraban inapropiadas las soluciones propuestas por Fox, tal vez porque este no conocía personalmente las condiciones particulares de la ciudad.

En este sentido, la presencia de compañías extranjeras rivalizaba fuertemente con los profesionales nacionales, pues generalmente los mayores contratos de la Nación eran adjudicados a firmas internacionales, que, por su mera procedencia, otorgaban prestigio a las obras (J.A.LI., 1927, p. 623) y representaban una garantía de éxito que en muchas ocasiones llevaba al gobierno a construir proyectos que no se adaptaban ni econonómica, ni geográfica, ni culturalmente a las necesidades del país.

Fox.propuso un sistema unitario de alcantarillas que consistía en recoger en un mismo colector las aguas lluvias y las servidas, tal como se encontraba planteado el alcantarillado de la ciudad en ese momento. Sin embargo, desestimó condiciones particulares como la recolección de las aguas que caían en la superficie de las calles y calculó el diámetro de las tuberías para una lluvia máxima de 35 milímetros por hora (pues le resultaba excepcional e imprevisto que pudieran presentarse lluvias más intensas), ignorando las condiciones metereológicas de la ciudad y poniendo en peligro los edificios, que, de acuerdo con los ingenieros Borda, Uribe y Garavito, podían terminar inundados en su parte baja (BORDA; URIBE; GARAVITO, 1912, p. 301).

Si bien el proyecto de Fox contemplaba detalles técnicos y medidas como llevar el colector hasta el desembocadero principal para no verter las aguas en los ríos San Francisco y San Agustín, no tuvo en cuenta recolectar las aguas que caían de los cerros y bajaban por las calles causando estragos y gran perjuicio en la ciudad. Así mismo, el desconocimiento de las condiciones particulares urbanas llevó al planteamiento de un sistema que debido a su poca inclinación no 
favorecía el destaponamiento de las tuberías mediante la corriente, lo cual también causaba problemas de funcionamiento de las alcantarillas.

Finalmente la casa Pearson $\delta$ Son hizo un presupuesto de las obras con base en el proyecto de Fox, el cual, aunque era minucioso en la enunciación de los detalles de las obras por hacer, resultaba de precios exagerados al contemplar costos que superaban hasta en más de cuatro veces los precios locales, por lo cual, se cuestionaba desde el gremio de profesionales colombianos la necesidad de encomendar estos trabajos a compañías extranjeras, que si bien imponían técnicas innovadoras para la implementación de servicios, provocaban gran despilfarro del dinero que el Estado podía invertir en la realización de mayor cantidad de obras dirigidas por los ingenieros nacionales (SANÍN, 1927, p. 633).

\section{Una aclaración necesaria sobre el norteamericano}

Uno de los mayores avances en el continente americano respecto al desarrollo de la electricidad, fue el alcanzado por Frederick Stark Pearson en Canadá, México, Brasil y Barcelona. Sin embargo, este ingeniero norteamericano no guarda ninguna relación con la compañía inglesa Pearson and Son y su trabajo en ambos continentes.

Nacido en Lowell, Massachusetts en $1861^{19}$, Fred Stark Pearson se graduó como ingeniero electricista de Tufts College en 1886 y desempeñó su primer trabajo en el subway de Nueva York, donde resultó ganador de un concurso para solucionar una de las líneas del transporte subterráneo y obtuvo el capital necesario para viajar a Brasil. Años más tarde regresaría al territorio brasileño, con el apoyo económico de empresarios canadiẹnses para fundar la São Paulo Tramway, Ligth and Power Companyen 1898 y cinco años después la Rio de Janeiro Tramway, Light and Power Co. (CODOY, 1996, p. 37), impulsando la electrificación del país suramericano. Posteriormente en México, Pearson lideró la construcción de la Hidroeléctrica de Necaxa construída por Mexican Light and Power Co. (PEÑA, 2012, p. 23-26) y participó a través de holding en la Mexico Tramways Co. y la Mexico North Western Railway Co. (CODOY, 1996, p. 37).

Antes de su viaje a Brasil, Frederick Pearson había participado en la construcción de las Centrales Hidroeléctricas de las Cataratas del Niágara, lugar donde se asoció financieramente con el

19 Frederick Stark Pearson murió a la edad de 54 años, cuando en 1915 el vapor Lusitania, en el que se desplazaba entre Nueva York y Lóndres, fue hundido durante la Primera Guerra Mundial (LA ELECTRICIDAD..., 2015). 
belga Alfred Loewenstein, con quien emprendería varias empresas dedicadas a la producción de energía y comunicaciones en Brasil, México y Barcelona (CAPEL, 1994, p. 18), de las cuales se destaca el holding Brazilian Traction Light and Power Company creado en 1912.

En 1911 Frederick constituyó en Canadá la Pearson Enginnering Company Ltd. que más tarde se transformó en Engineering Corporation Ltd. y agrupó una serie de compañías dedicadas a la producción de electricidad, construcción de ferrocarriles y líneas de tranvía, proyectos de regadío, gas e ingeniería en general (CAPEL, 1994, p. 18), destacándose la electrificación de Cataluña conocida como La Canadiense (CAPEL, 1994, p. 25), la cual daría origen a la Barcelona Traction Light \& Power Co. Ltd. y su filial Ebro Irrigation \& Power Co. Ltd.

Es necesario diferenciar el trabajo simultáneo de las dos compañías lideradas por ingenieros de apellido Pearson. Mientras los ingleses de S. Pearson and Son trabajaron en la construcción de obras de saneamiento y suministro de agua en varios países americanos, el ingeniero norteamericano Fred Stark Pearson desarrolló obras de electrificación en América y Cataluña, coincidiendo ambas compañías en países como Canadá, Estados Unidos y México, sin evidencia de que hayan realizado proyectos comunes.

\section{Conclusiones}

Aunque los conceptos de higiene se empezaron a popularizar desde la medicina y tuvieron su mayor desarrollo durante el siglo XIX, fue en los inicios del siglo XX que se avanzó en la implementación de normas para higienizar las ciudades y poner en práctica los adelantos industriales mediante el ejercicio de la ingeniería, modernizando las urbes y transformándolas en ambientes higiénicamente dispuestos. Así, el crecimiento urbano se empezó a regir por conceptos de orden y saneamiento que convirtieron las redes de agua en herramientas de control social y desarrollo, determinando con su cobertura, la dirección y expansión de la ciudad.

El poder de la técnica se vió representado en los conceptos de habitabilidad y confort urbano, imponiendo el saneamiento por medio de la construcción de infraestructuras que buscaban facilitar y promover la vida urbana en condiciones higiénicas. En este sentido, el papel de las compañías extranjeras fue determinate para introducir normas y nuevas tecnologías desarrolladas en Europa y Norteamérica. A partir del conocimiento científico, el aporte de los ingenieros fue determinante para el desarrollo urbano, no solo por la elaboración técnica de los 
proyectos, sino por el trasvase de conocimientos que derivó en el avance de las infraestructuras y la homogenizacíón de los mercados, llevando a una rápida industrialización de las obras.

El ingeniero británico surge en un ambiente de intensa actividad industrial, artesanos-, inventores-emprendedores hacen gala de una enorme creatividad técnica. No existe una enseñanza reglada del arte del ingeniero, sino que el aprendizaje se realiza en el taller, bajo la tutela de maestros; es decir, el esquema de transmisión del conocimiento es aún, esencialmente, de tipo gremial. (SILVA, 2007, p. 46)

Las compañías inglesas fueron pioneras en el manejo de las redes de agua y las infraestructuras asociadas a su desarrollo, lo cual derivó no solo en la construcción de proyectos a gran escala, sino en el avance de las obras públicas a partir de la elaboración de planos, especificaciones técnicas, presupuestos detallados y proyecciones futuras para las obras urbanas, marcando la presentación técnica de las propuestas y la previsión del alcance y cobertura de los proyectos. Sin embargo, representaban para las naciones latinoamericanas grandes inversiones de dinero con el fin de cubrir los costos de honorarios y desplazamientos del personal requerido por cada compañía.

La tecnificación de la ciudad representó el crecimiento y desarrollo de la estructura urbana en las primeras décadas del siglo XX, pues se implementaron procesos que permitieron sortear condiciones topográficas y llevaron progreso a nuevas zonas de urbanización, ampliando las áreas de expansión. Con la tecnificación de los procesos y la construcciones de redes de servicios, las ciudades se modernizaron en busca de brindar confort y un estilo de vida asociado al modelo de progreso, convirtiendo el crecimiento urbano de este periodo, en un proceso que dejó atrás las ciudades coloniales y los sistemas artesanales para introducir los avances tecnologicos, representando un salto hacía la metropolización urbana por medio de la implementación de redes que representaron tanto el ordenamiento de las estructuras urbanas y administrativas, como la exclusión de zonas que quedaron marcadas por el subdesarrollo.

\section{Referencias}

ALCAIDE, Rafael. La introducción y el desarrollo del higienismo en España durante el siglo XIX. Precursores, continuadores y marco legal de un proyecto científico y social. Scripta Nova. Revista electrónica de Geografiay Ciencias Sociales, Barcelona, n. 3, p. 32-54, 1999. Disponible en: <https://dialnet.unirioja.es/ servlet $/$ articulo? codigo $=245050>$. Acceso en: 8 oct. 2019. 
BORDA, Alberto; URIBE, Pedro; GARAVITO, Julio. Informe Ingeniería Municipal. Anales de Ingeniería, Bogotá, p. 227-228, 300-304, enero/feb. 1912.

CAPEL,Horacio. El agua comoservicio público.A propósito delSeminariolnternacional Faire parlerles reseaux: Leau, Europe-Amérique Latine. Biblio 3W. Revista Bibliográfica de Ceografia y Ciencias Sociales, Barcelona, v. 5, n. 218, 2000. Disponible en: <http://www.ub.edu/geocrit/b3w-218.htm> Acceso en: 8 oct. 2019.

CAPEL, Horacio. Las tres chimeneas. Barcelona: FECSA, 1994.

CASAS, Álvaro. Los circuitos del agua y la higiene urbana en la ciudad de Cartagena a comienzos del siglo XX. História, Ciências, Saúde - Manguinhos, Rio de Janeiro, v. 7, n. 2, p. 349-377, out. 2000. Disponible en: <http:// dx.doi.org/10.1590/S0104-59702000000300006> Acceso en: 8 oct. 2019.

DUPUY, Gabriel. El urbanismo de las redes. Teorías y métodos. Barcelona: Oikos-Tau, 1998.

EL INCENIERO Eduardo Budge y los 100 años del Puerto de Valparaíso. Mundo marítimo, Santiago, p. 7, 7 enero 2013. Disponible en: <https://www.mundomaritimo.cl/noticias/el-ingeniero-eduardo-budge-y-los100-anos-del-puerto-de-valparaiso>. Acceso en: 8 oct. 2019.

FRISCHMANN, Brett. Infraestructure. New York: Oxford University Press, 2012.

GODOY, Ernesto. Un ingeniero y su imperio: Frederick Stark Pearson. Revista de la Universidad de México, México, n. 545, p. 35-39, jun.1996. Disponible en: <https://www.revistadelauniversidad.mx/articles-files/134d0e12c3b4-481b-b352-e3b45f3d3041>. Acceso en: 8 oct. 2019.

HENDERSON, James. La modernización en Colombia. Los años de Laureano Gómez 1889-1965. Medellín: Editorial Universidad de Antioquia, 2006.

J.A.LI. Los contratos de construcción y los ingenieros nacionales. Anales de Ingeniería, Bogotá, n. 416, p. 623625 , nov. 1927.

LA CONCESIÓN del ferrocarril de Tehuantepec a la Pearson and Son Limited de Londres. El Correo de México, México, 6 oct. 1906. (Colección Documentos Porfiristas, Biblioteca Aportación Histórica, 1948).

LA ELECTRICIDAD del doctor Pearson. El Periódico, Barcelona, 3 mayo 2015. Disponible en: <https://www. elperiodico.com/es/internacional/20150501/la-electricidad-del-doctor-pearson-4152072>. Acceso en: 8 out. 2019.

LAFUENTE, Antonio. El ensanche de la ciencia (1834-1936). In: CRESPO, Virgilio Pinto (Ed.). Atlas histórico de la ciudad de Madrid (1850-1939). Madrid: Lunwerg Editores, 2001. p. 140-169. Disponible en: <https://www.academia. edu/4845898/El_ensanche_de_la_ciencia_1834-1936>. Acceso en: 8 oct. 2019.

PEARSON AND SON. Grace's Guide to British Industrial History. Disponible en: <https://www.gracesguide. co.uk/S._Pearson_and_Son>Acceso en: 8 oct. 2019.

PEARSON PLC. Company profile, information, business description, history, background information on Pearson PLC. Disponible en: <www.referenceforbusiness.com/history2/38/PEARSON-PLC.html>. Acceso en: 8 oct. 2019. 
PEÑA, Celina. Frederick Stark Pearson y la construcción de la hidroeléctrica de Necaxa. In: SIMPOSIO INTERNACIONAL GLOBALIZACIÓN, INNOVACIÓN Y CONSTRUCCIÓN DE REDES TÉCNICAS URBANAS EN AMÉRICA Y EUROPA, 1890-1930. BRAZILIAN TRACTION, BARCELONA TRACTION Y OTROS CONGLOMERADOS FINANCIEROS Y TÉCNICOS, 2012, Barcelona. Actas... Barcelona: Universidad de Barcelona, Facultad de Ceografía e Historia, 2012. p. 23-26. Disponible en: <http://www.ub.edu/geocrit/ Simposio/cPe\%C3\%B1a_Frederick.pdf . Acceso en: 10 dic. 2019.

RAMOS, José. Edwin Chadwick, el movimiento británico de salud pública y el higienismo español. Revista de historia industrial, Barcelona, n. 55, p. 11-38, 2014. Disponible en: <http://www.raco.cat/index.php/ Historialndustrial/article/view/280098>. Acceso en: 8 oct. 2019.

RUEDA, José. Madrid en torno a 1898: información y gestión urbana (higienismo y reforma municipal). Historia y comunicación social, Madrid, n. 3, p. 177-194, 1998. Disponible en: <https://revistas.ucm.es/index. php/HICS/article/viewFile/HICS9898110177A/19786>. Acceso en: 8 oct. 2019.

SAMBRICIO, Carlos. De ciudad-capital a metrópolis. La génesis de un nuevo modelo urbano. La influencia del urbanismo alemán en la España del primer cuarto del siglo XX. In: BAUDER, Eve. Berlín-Madrid: arquitecturas endiálogo.Madrid: Embajada de la República Federal de Alemania, 2017. p.196-217. Disponible en:<file://C:/ Users/ap470100/Downloads/De_ciudad-capital_a_Metropolis._La_genes.pdfs. Acceso en: 8 oct. 2019.

SANÍN, C. Grandes contratos para obras públicas. Anales de Ingeniería, Bogotá, n. 416, p. 632-634, nov. 1927.

SILVA, Manuel. El Ochocientos: de la involución postilustrada y la reconstrucción burguesa. In: SILVA, Manuel (Ed.). Técnica e ingeniería en España. El Ochocientos: pensamiento, profesiones y sociedad. Zaragoza: Real Academia de Ingeniería; Institución Fernando el Católico; Prensas Universitarias, 2007. p. 9-104.

SUÁREZ, Miguel; MONSALVO, Edwin. La higiene y el progreso. La institucionalización de la burocracia sanitaria en Manizales.1920-1940. Anuario de historia regionaly de las fronteras, Bucaramanga, v. 18, n. 1, p. 99125, jun. 2013.

VALLEJO, Gustavo. Higienismo y sectores populares en La Plata. 1882-1910. Estudios del hábitat, Buenos Aires, v. 2, n. 5, p. $57-72,1997$.

VARIAS leyes importantes. Anales de Ingeniería, Bogotá, n. 283-284, p.113-117, sept./oct. 1916. Sección Editorial.

Recebido em: 13 de agosto de 2019 Aprovado em: 21 de setembro de 2019 